\title{
Nutation Spectroscopy of a Nanomagnet Driven into Deeply Nonlinear Ferromagnetic Resonance
}

\author{
Y. Li ${ }^{1,}{ }^{*}$ V. V. Naletov, ${ }^{1,2}$ O. Klein, ${ }^{3}$ J. L. Prieto, ${ }^{4}$ M. Muñoz, ${ }^{5}$ V. Cros, ${ }^{6}$ P. Bortolotti,${ }^{6}$ \\ A. Anane, ${ }^{6}$ C. Serpico, ${ }^{7}$ and G. de Loubens ${ }^{1, \dagger}$ \\ ${ }^{1}$ Service de Physique de l'État Condensé, CEA, CNRS, Université Paris-Saclay, \\ 91191 Gif-sur-Yvette, France \\ ${ }^{2}$ Institute of Physics, Kazan Federal University, 420008 Kazan, Russian Federation \\ ${ }^{3}$ Université Grenoble Alpes, CEA, CNRS, Grenoble INP, INAC-Spintec, 38000 Grenoble, France \\ ${ }^{4}$ Instituto de Sistemas Optoelectrónicos y Microtecnología (UPM), 28040 Madrid, Spain \\ ${ }^{5}$ Instituto de Micro y Nanotecnología (CNM-CSIC), 28760 Madrid, Spain \\ ${ }^{6}$ Unité Mixte de Physique CNRS, Thales, Université Paris-Sud, Université Paris-Saclay, \\ 91767 Palaiseau, France \\ ${ }^{7}$ Dipartimento di Ingegneria Elettrica e Tecnologie dell'Informazione, \\ Università Federico II, 80138 Napoli, Italy
}

(Received 9 April 2019; revised manuscript received 4 September 2019; published 19 November 2019)

Strongly out-of-equilibrium regimes in magnetic nanostructures exhibit novel properties, linked to the nonlinear nature of magnetization dynamics, which are of great fundamental and practical interest. Here, we demonstrate that ferromagnetic resonance driven by microwave magnetic fields can occur with substantial spatial coherency at an unprecedented large angle of magnetization precessions, which is normally prevented by the onset of spin-wave instabilities and magnetization turbulent dynamics. Our results show that this limitation can be overcome in nanomagnets, where the geometric confinement drastically reduces the density of spin-wave modes. When the obtained deeply nonlinear ferromagnetic resonance regime is perturbed, the magnetization undergoes eigenoscillations around the steady state due to torques tending to restore the stable large-angle periodic trajectory. These eigenoscillations are substantially different from the usual spin-wave modes around the ground state because their existence is connected to the presence of a large coherent precession. They are experimentally investigated by a new spectroscopic technique based on the application of a second microwave excitation field that is tuned to resonantly drive them. This two-tone spectroscopy enables us to show that they consist in slow coherent magnetization nutations around the large-angle steady precession, whose frequencies are set by the balance of restoring torques. Our experimental findings are well accounted for by an analytical model derived for systems with uniaxial symmetry. They also provide a new means for controlling highly nonlinear magnetization dynamics in nanostructures, opening interesting applicative opportunities in the context of magnetic nanotechnologies.

DOI: 10.1103/PhysRevX.9.041036

\section{INTRODUCTION}

Spectroscopy based on the resonant interaction of electromagnetic fields with material media has had a tremendous impact on the development of physics since the beginning of the 20th century and remains of crucial

\footnotetext{
*Present address: Materials Science Division, Argonne National Laboratory, Argonne, Illinois, USA.

${ }^{\dagger}$ gregoire.deloubens@cea.fr

Published by the American Physical Society under the terms of the Creative Commons Attribution 4.0 International license. Further distribution of this work must maintain attribution to the author(s) and the published article's title, journal citation, and DOI.
}

Subject Areas: Condensed Matter Physics, Magnetism, Nonlinear Dynamics importance in the study of nanotechnologies. In this area, a central role is played by magnetic resonance spectroscopy, which includes various techniques such as nuclear magnetic resonance (NMR), electron paramagnetic resonance (EPR), and ferromagnetic resonance (FMR), all based on the excitation of the Larmor precession of magnetic moments around their equilibrium position [1].

FMR differs from NMR and EPR in the fact that in ferromagnetic media, magnetic moments are coupled by strong exchange interactions that tend to align them, leading to a large macroscopic spontaneous magnetization. In these conditions, magnetodipolar effects become important and determine large internal fields that enrich both the ground state-which can be spatially nonuniform —and the dynamics of magnetic moments. The complex interactions 
taking place in the media can be described by an appropriate effective field that sets the timescale of the magnetization precession and that itself depends on the magnetization, making the dynamics, for sufficiently large deviations from the ground state, highly nonlinear [2]. A special role in FMR is also played by the spin waves (SWs), which are the collective eigenmodes associated with small magnetization oscillations around the equilibrium configuration [3]. When pumping fields excite SWs well above their thermal amplitudes, a rich variety of phenomena emerges, such as the formation of dynamical solitons [4,5], SW turbulences and chaos [4,6-8], and Bose-Einstein condensation of magnons [9], the quanta of SWs.

Recent developments in magnetic nanotechnologies have also demonstrated that FMR and SW dynamics can be excited either by microwave magnetic fields or by spin transfer torques, with the promise of innovative magnonic and spintronic devices for information and communication technologies [10]. In this area, spin torque nano-oscillators (STNOs) [11-15], which exhibit strong nonlinear properties [16], have even been successfully implemented to perform neuromorphic tasks $[17,18]$.

The complexity of magnetization dynamics when strongly nonlinear regimes set in is usually detrimental to the reliable control of nanomagnetic devices, such as oscillators, memories, and logic gates. In this respect, it is important to establish how far from equilibrium magnetic nanostructures can be driven before the coherent magnetization dynamics becomes highly perturbed by the onset of SW instabilities [19]. In this article, we provide a crucial advancement for this problem. We demonstrate that FMR in a sufficiently confined nanostructure can exhibit unprecedented large-angle magnetization precessions that are spatially quasiuniform. The experimental evidence of the coherence of large precessions is brought about by a new spectroscopic technique based on the application of a second probe excitation field, with frequency close to the one of the main time-harmonic field. This second excitation is used to drive small eigenoscillations of magnetization around the FMR large-angle periodic oscillations, corresponding to coherent nutations of the magnetization. These nutation modes are substantially different from the usual SW modes around the ground state because they correspond to eigenmodes around a far-fromequilibrium state. Their existence is connected to the one of a large coherent precession, and their frequencies are set by the balance of restoring torques acting on the magnetization when it is perturbed from its large-angle periodic trajectory. Moreover, we show that the resonant excitation of these nutations can be used to control the nonlinear magnetization dynamics by affecting the switching fields associated with the bistability of the large-angle FMR response pictured in Fig. 1(a), which occurs beyond the foldover instability predicted by Anderson and Suhl [20].

\section{RESULTS}

In the following, we investigate the FMR of an individual nanodisc of yttrium iron garnet (YIG) in the perpendicular configuration. The choice of YIG is natural as it is the magnetic material with the smallest SW damping, making it attractive to study weakly dissipative magnetization dynamics in the linear and nonlinear regimes [21]. The nanodisc has a diameter of $700 \mathrm{~nm}$ and is patterned from a 20-nm-thick YIG film of magnetization $\mu_{0} M_{s}=0.21 \mathrm{~T}$ [22], $\mu_{0}$ being the vacuum permeability. It is saturated out of plane by a magnetic field $\boldsymbol{H}_{0}$ applied along its normal, $z$. A broadband antenna supplies a spatially uniform, linearly polarized microwave field of pulsation $\omega_{1}$ oriented in the plane of the nanodisc. It can be decomposed into its left and right circularly polarized components, with only the latter being efficiently coupled to the Larmor precession of the magnetization. In the following, $h_{1}$ will refer to the circular amplitude of the excitation field produced by the output power $P_{1}$ from the synthesizer. It drives the YIG nanodisc into FMR, thereby opening a precession angle $\theta$ of the magnetization $\boldsymbol{M}$ around $\boldsymbol{H}_{0}$ and decreasing its longitudinal component $M_{z}=M_{s} \cos \theta$. This dynamics is characterized by magnetic resonance force microscopy (MRFM), which sensitively probes the variation $\Delta M_{z}=M_{s}-M_{z}$ through the dipolar force between the YIG nanodisc and a magnetic nanosphere attached at the end of a soft cantilever [23], as sketched in Fig. 1(b). Further details about the sample, the MRFM setup, and the microwave calibration can be found in the Appendix A.

\section{A. Linear spin-wave spectroscopy}

In Fig. 1(c), the SW spectroscopy of the YIG nanodisc is performed at $\omega_{1} /(2 \pi)=10.5 \mathrm{GHz}$ and low power $P_{1}=-30 \mathrm{dBm}$, which is pulse modulated at the frequency of the MRFM cantilever, $f_{c} \approx 12.3 \mathrm{kHz}$, to improve the signal-to-noise ratio (the FMR signal is coded in the amplitude of the cantilever vibrations; see Appendix A 2). Quantized radial SW modes are excited by the uniform pumping field [24]. Their spatial profiles indexed by the radial number are shown above the spectrum. The fundamental Kittel mode is the one excited at the largest field, $\mu_{0} H_{K}=0.569 \mathrm{~T}$, and it corresponds to a uniform phase of the transverse magnetization in the disc. Because of the geometric confinement, it is well separated from other SW modes at lower fields [25]. Its full width at half maximum, $\mu_{0} \Delta H=0.35 \mathrm{mT}$, is determined at even lower power $\left(P_{1}=-38 \mathrm{dBm}\right)$ to avoid distortions of the resonance line due to the onset of foldover, which occurs when the change in the effective field becomes comparable to the FMR linewidth, at $P_{1}^{\text {th }}=-33 \mathrm{dBm}$ or $\mu_{0} h_{1}^{\text {th }}=0.009 \mathrm{mT}$ (see Appendix A 3). It corresponds to a Gilbert damping parameter $\alpha=\gamma \mu_{0} \Delta H /\left(2 \omega_{1}\right)=4.7 \times 10^{-4}$, where $\gamma$ is the gyromagnetic ratio, in agreement with the value determined 
(a)

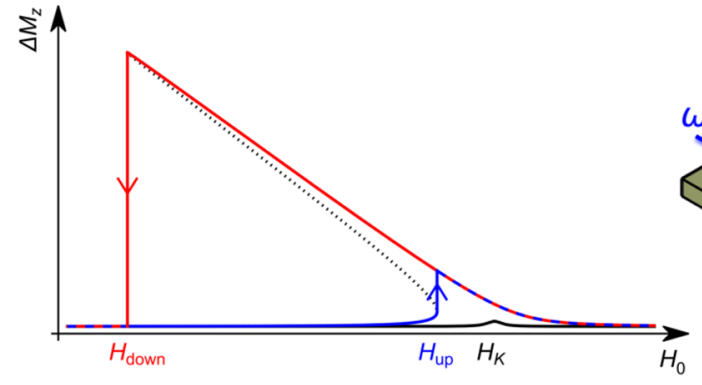

(c)

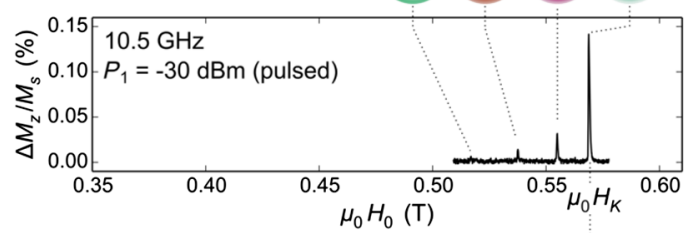

(d)

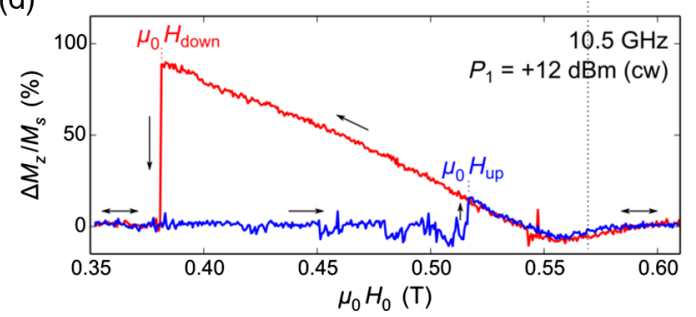

(b)

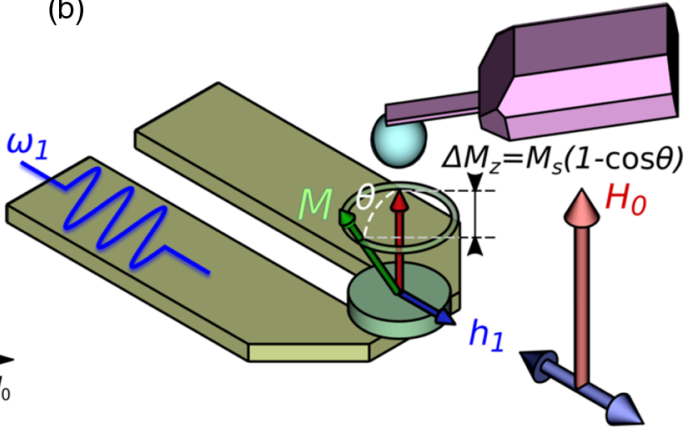

(e)

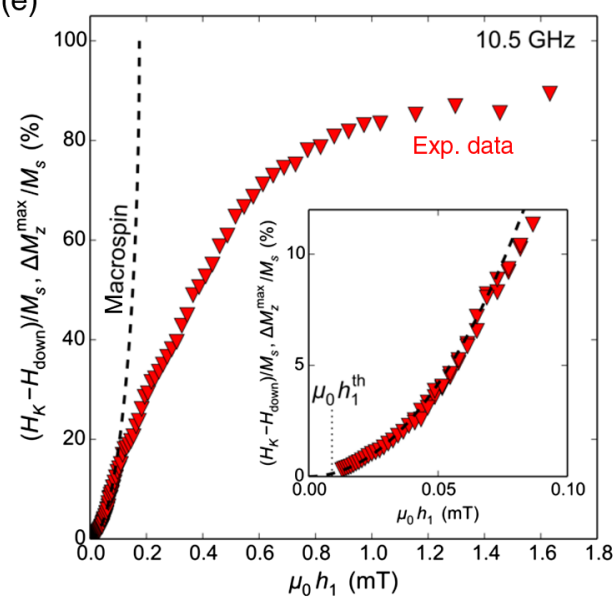

FIG. 1. Ultrastrong foldover of FMR. (a) Illustration of hysteretic foldover in the nonlinear regime of FMR, where jumps between the two stable branches of the dynamics occur at $H_{\text {down }}$ and $H_{\text {up }}$. The dotted line is the unstable branch. The black Lorentzian curve centered at $H_{K}$ corresponds to linear FMR. (b) Schematics of the experiment. A microwave field $\boldsymbol{h}_{1}$ of pulsation $\omega_{1}$ drives the magnetization $\boldsymbol{M}$ of a YIG nanodisc into FMR, opening a precession angle $\theta$ around the perpendicularly applied field $\boldsymbol{H}_{0}$. The associated variation in the longitudinal component of the magnetization, $\Delta M_{z}$, is mechanically detected by the cantilever of a magnetic resonance force microscope. (c) Spin-wave spectroscopy performed at $10.5 \mathrm{GHz}$ in the linear regime. The profiles of the quantized radial SW modes, calculated using a micromagnetic code, are shown above (different colors display regions precessing in opposite phase). (d) FMR spectrum in the deeply nonlinear regime exhibiting ultrastrong hysteretic foldover and nearly complete suppression of $M_{z}$. (e) Evolution of the maximal normalized foldover shift as a function of the pumping field $h_{1}$. The dashed line shows the behavior expected for a macrospin, and the inset is a zoomed view of the low-amplitude regime.

from broadband measurements (see Supplemental Material, Fig. S1 [26]).

\section{B. Deeply nonlinear FMR}

The FMR spectrum of the YIG nanodisc radically changes at much stronger pumping fields. Figure 1(d) shows the measurement with a continuous wave (cw) excitation at $P_{1}=+12 \mathrm{dBm}$, i.e., more than 4 orders of magnitude larger than the threshold of foldover instability. The cw excitation allows us to reveal the bistable character of the nonlinear magnetization dynamics (the FMR signal is now coded in the cantilever frequency; see Appendix A 2). By sweeping down the applied field (red curve) through the resonance of the Kittel mode, the precession angle substantially increases, which decreases the static demagnetizing field $\mu_{0} M_{z}$ and shifts the FMR condition $\omega_{1}=\gamma \mu_{0}\left(H_{0}-M_{z}\right)$ to a lower magnetic field by the same amount. This foldover shift towards low fields continues until the pumping field cannot sustain the largeamplitude magnetization dynamics anymore, causing the sharp downward jump to the lower stable branch observed at $\mu_{0} H_{\text {down }}=0.381 \mathrm{~T}$. By sweeping up the applied field (blue curve), an upward jump to the higher stable branch is observed at $\mu_{0} H_{\text {up }}=0.516 \mathrm{~T}$. The extremely hysteretic foldover witnessed in this experiment is remarkable, if one compares it to the much weaker bistable response characteristics observed before [27,28]. Moreover, the maximal foldover shift $\mu_{0}\left(H_{K}-H_{\text {down }}\right)=0.188 \mathrm{~T}$ corresponds to a reduction of nearly $90 \%$ of $\mu_{0} M_{z}$ induced by the microwave pumping, which translates into a mean precession angle of $84^{\circ}$ in the nanodisc.

The evolution of the maximal normalized foldover shift as a function of the pumping field $h_{1}$ is plotted in Fig. 1(e) together with $\Delta M_{z}^{\max } / M_{s}=1-\sqrt{1-4 h_{1}^{2} / \Delta H^{2}}$ calculated from the macrospin Landau-Lifshitz-Gilbert (LLG) equation [3]. The measured foldover shift starts to deviate 
from the macrospin model beyond $\mu_{0} h_{1} \simeq 0.1 \mathrm{mT}$, which is an order of magnitude larger than the threshold for foldover, when the angle of the uniform precession increases above $30^{\circ}$, corresponding to $\Delta M_{z} / M_{s} \simeq 15 \%$. This result is the signature of the onset of SW instabilities [19], which is significantly postponed compared to what is observed in larger YIG samples, where the Suhl threshold is reached even before the onset of foldover, for a uniform precession angle of only a couple of degrees [29,30]. In this respect, the experimental results presented in Fig. 1 demonstrate that the discretization of the excitation spectrum in nanostructures efficiently inhibits nonlinear interactions between SW modes, which enables the preservation of coherent magnetization dynamics to much higher power levels $[31,32]$.

\section{Nutation spectroscopy in the rotating frame}

We now aim at further probing the large-amplitude magnetization dynamics demonstrated above, which is periodic at $\omega_{1}$ in the laboratory frame and hence referred to as a $\mathbf{P}$-mode [33]. In the frame rotating with $\boldsymbol{h}_{1}$ at $\omega_{1}$ around the $z$ axis, the magnetization $\boldsymbol{M}_{0}$ of a $\mathbf{P}$-mode is fixed at a polar angle $\theta_{0}$ and a phase lag $\varphi_{0}$ [see Fig. 2(a) and Supplemental Material [26] ]. For this case, we conduct two-tone measurements, where in addition to the strong $\mathrm{cw}$ excitation $\boldsymbol{h}_{1}$ at $\omega_{1}$, a second weak microwave field $\boldsymbol{h}_{2}$, that is pulse modulated at the cantilever frequency $f_{c}$ to improve the sensitivity, is applied at $\omega_{2}$, as shown in Fig. 2(b). MRFM is used to simultaneously detect $\Delta M_{1}$ induced by the main $\mathrm{cw}$ pumping at $\omega_{1}$ [by monitoring the cantilever frequency, as in Fig. 1(d)] and the additional change in longitudinal magnetization, $\Delta M_{2}$, induced by the second excitation at $\omega_{2}$ [by monitoring the amplitude of the cantilever vibrations, as in Fig. 1(c)]. The former informs us about the time-harmonic steady-state regime driven by $\boldsymbol{h}_{1}$, whereas the latter allows us to spectroscopically probe the eigenexcitations on top of this $\mathbf{P}$-mode. The $\Delta M_{2}$ spectrum measured at constant bias field $\mu_{0} H_{0}=$ 0.52 T by sweeping $\omega_{2} /(2 \pi)$ at low power $P_{2}=-16 \mathrm{dBm}$ in the vicinity of the frequency $\omega_{1} /(2 \pi)=10.5 \mathrm{GHz}$ of the main pumping $\left(P_{1}=+8 \mathrm{dBm}\right)$ is shown in Fig. 2(d). It displays two narrow resonance peaks centered at 10.2 $\mathrm{GHz}$ and $10.8 \mathrm{GHz}$, i.e., symmetrically with respect to $\omega_{1} /(2 \pi)$, which means that in the frame rotating with $\boldsymbol{h}_{1}$ at $\omega_{1}$, the magnetization is precessing at $\left(\omega_{2}-\omega_{1}\right) /(2 \pi)=$ $\pm \omega_{\mathrm{P}, 0} /(2 \pi)= \pm 0.3 \mathrm{GHz}$ around its equilibrium position $\boldsymbol{M}_{0}$ [cf. Fig. 2(a)]. In other words, it is subjected to a slow nutation motion in the laboratory frame: a combination of the large periodic motion at frequency $\omega_{1}$ and the small oscillations at frequency $\omega_{\mathrm{P}, 0}<\omega_{1}$. The dependence of the nutation frequency $\omega_{\mathrm{P}, 0}$ on the main pumping field $h_{1}$ at fixed $\mu_{0} H_{0}=0.52 \mathrm{~T}$ is presented in Fig. 2(e), whereas its evolution measured as a function of the down-swept field $H_{0}$ at fixed $P_{1}=+8 \mathrm{dBm}$ and $P_{1}=+1 \mathrm{dBm}$ is shown in the 2D spectroscopy maps of Figs. 2(f) and 2(h), respectively.

From the framework of dynamical systems [2], it is known that when the $\mathbf{P}$-mode is stable and of focus type, the relaxation dynamics toward the $\mathbf{P}$-mode, from small perturbations from the $\mathbf{P}$-mode, is a slowly damped oscillation. Here, the eigenfrequency of the corresponding nutation dynamics is set by two restoring torques. The first one is directly provided by $\boldsymbol{h}_{1}$, which sets the Rabi frequency in a magnetic resonance experiment [34,35]. It depends both on the phase $\varphi_{0}$ between the magnetization and the driving field and on the precession cone angle $\theta_{0}$. The second one is a demagnetizing torque specific to nonlinear FMR, which only depends on $\theta_{0}$ and stiffens the nutation resonance. Therefore, the low-frequency nutation dynamics discussed herein is fundamentally distinct from the one possibly occurring at very high frequency due to an additional inertial torque, which might be present in the equation of motion of the magnetization [36-38]. Following the theoretical approach of Ref. [39], it is possible to analytically calculate the frequency $\omega_{\mathrm{P}, 0}$ of spatially uniform nutation around a given $\mathbf{P}$-mode based on the macrospin LLG equation. Technical details are given in Appendix B (the full derivation is presented in the Supplemental Material [26]). In the limit of small damping, $\alpha \ll 1$, it can be expressed as a function of $h_{1}$ and the angles $\theta_{0}$ and $\varphi_{0}$ of the $\mathbf{P}$-mode as follows:

$$
\frac{\omega_{\mathrm{P}, 0}^{2}}{\gamma^{2}}=\frac{\mu_{0} h_{1} \cos \varphi_{0}}{\sin \theta_{0}}\left(\frac{\mu_{0} h_{1} \cos \varphi_{0}}{\sin \theta_{0}}+\mu_{0} M_{s} \sin ^{2} \theta_{0}\right) .
$$

This analytical expression is plotted as black dashed lines in Figs. 2(e), 2(f), and 2(h), using the amplitude $h_{1}$ of the main driving field in the experiments, the angle of precession at the bias field $H_{0}$ determined from the normalized foldover shift, $\cos \theta_{0}=\left(H_{K}-H_{0}\right) / M_{s}$, and the phase lag, which satisfies $\gamma \mu_{0} h_{1} \sin \varphi_{0}=\alpha \omega_{1} \sin \theta_{0}$ in the macrospin model. It reproduces rather well the experimental data, except in regions where the level of excitation is very large, due to the deviation from the macrospin behavior already noticed in Fig. 1(e). In addition, we have conducted full micromagnetic simulations in the time domain (see Appendix C), which allow us to extract the nutation frequency from the relaxation of the magnetization towards the steady-state regime driven by $\boldsymbol{h}_{1}$, shown in Fig. 2(c). The obtained results are plotted as red dotted lines in Figs. 2(e), 2(f), and 2(h). They quantitatively agree with the data on the full range of parameters investigated because, contrary to the macrospin approach, micromagnetic simulations can capture the SW instabilities occurring at high amplitude in the experiments, which slightly renormalize the nutation frequency (see discussion in Sec. III).

The dependence of the nutation frequency on $h_{1}$ observed in Fig. 2(e) can be explained as follows. There 
(a)
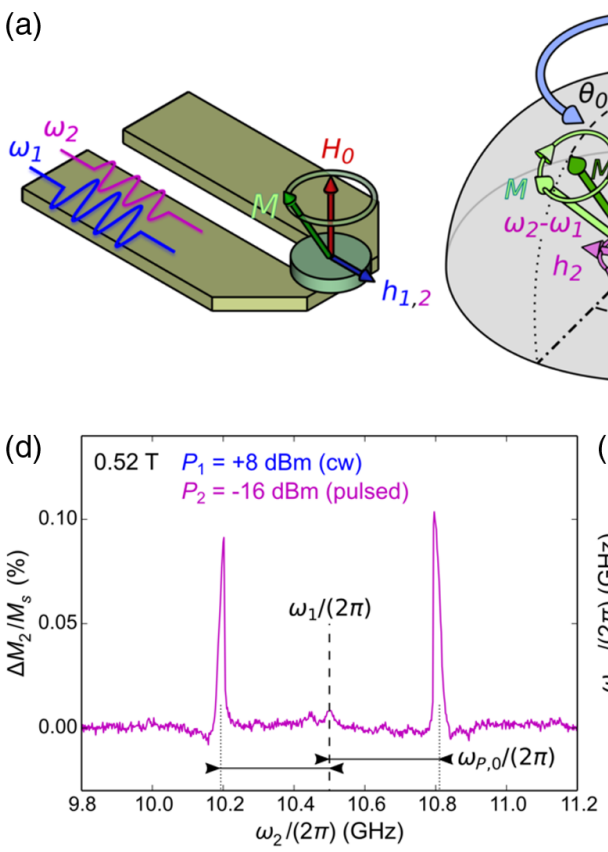

(d)

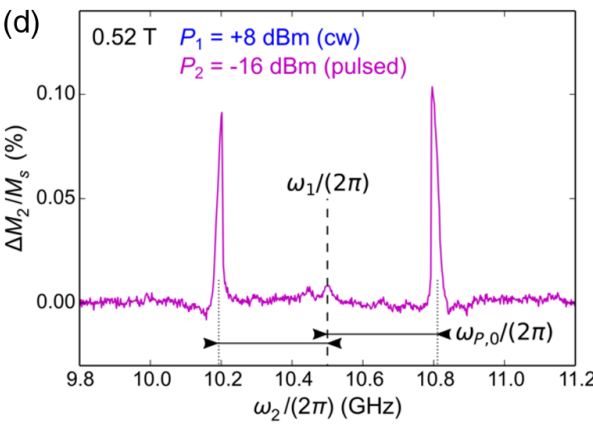

(f)

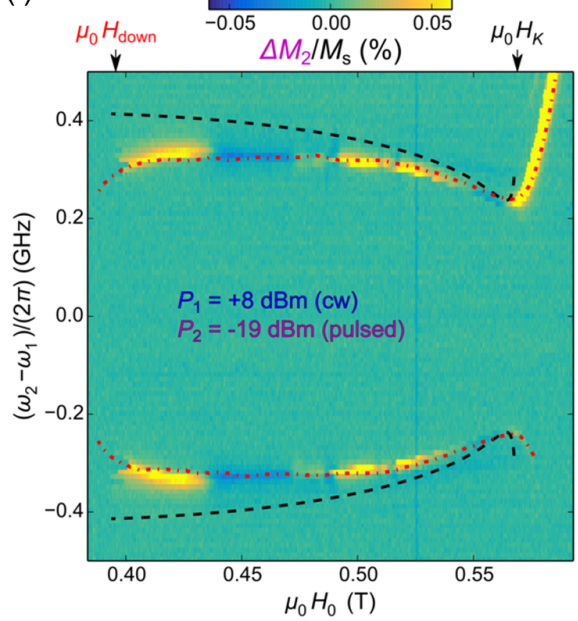

(h)

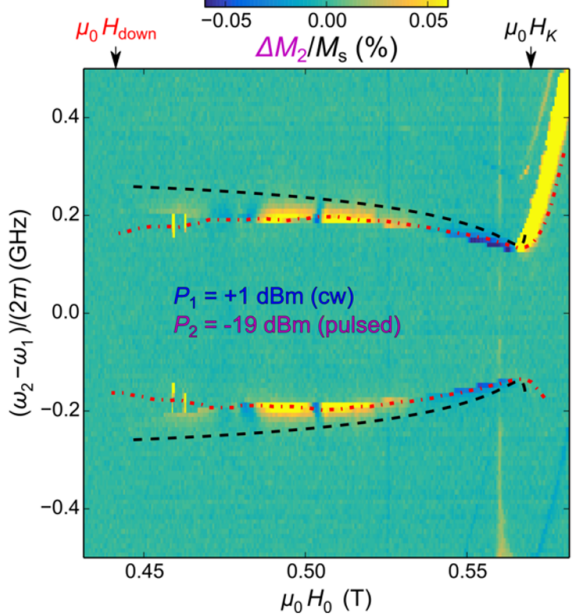

(b)

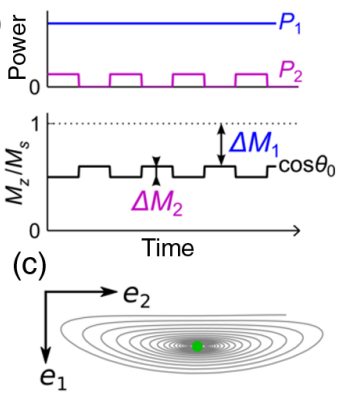

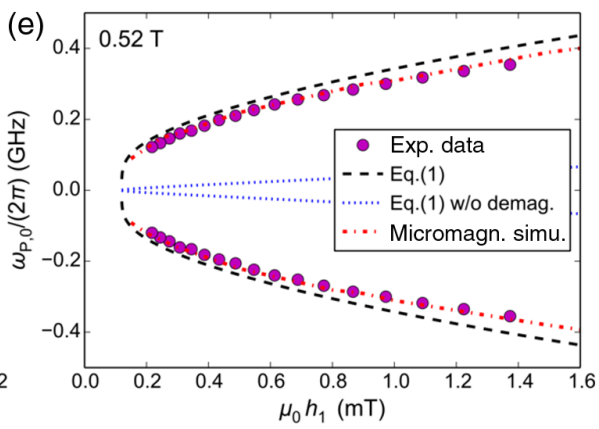

(g)
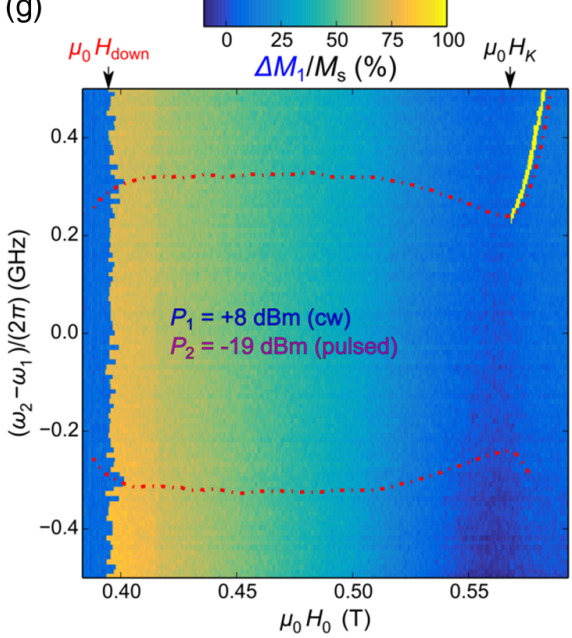

(i)

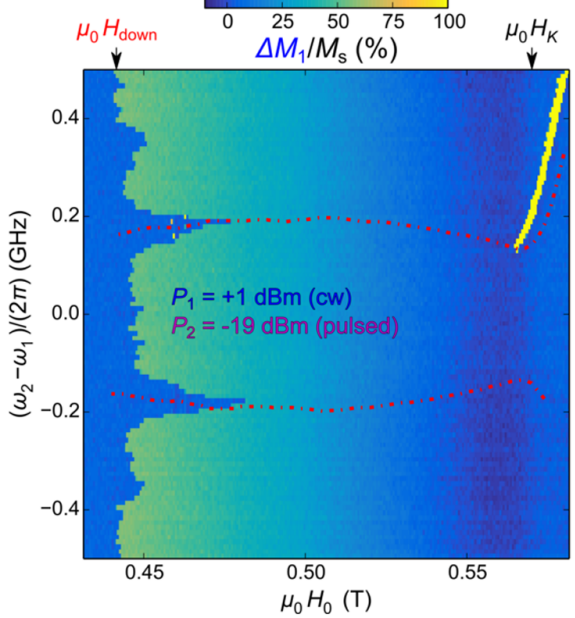

FIG. 2. (Continued) 
FIG. 2. Nutation of magnetization. (a,b) Principle of the experiment. A low-power microwave field $\boldsymbol{h}_{2}$ of pulsation $\omega_{2}$, which is pulse modulated at the cantilever frequency $f_{c} \approx 12.3 \mathrm{kHz}$, is added to the main pumping cw field $\boldsymbol{h}_{1}$. This addition enables the spectroscopy in the frame rotating with $\boldsymbol{h}_{1}$ at $\omega_{1}$, where the magnetization $\boldsymbol{M}_{0}$ of the $\mathbf{P}$-mode is fixed at an angle $\theta_{0}$ and a phase lag $\varphi_{0}$. (c) Relaxation trajectory of the magnetization towards the $\mathbf{P}$-mode in the plane $\left(\boldsymbol{e}_{1}, \boldsymbol{e}_{2}\right)$ orthogonal to $\boldsymbol{M}_{0}$ defined in Appendix B, calculated using micromagnetic simulations. (d) Spectroscopy performed at $\omega_{1} /(2 \pi)=10.5 \mathrm{GHz}$ and $\mu_{0} H_{0}=0.52 \mathrm{~T}$ as a function of $\omega_{2}$. The two resonance peaks that are symmetric with respect to $\omega_{1}$ correspond to a motion of nutation in the laboratory frame. (e) Evolution of the nutation frequency as a function of the main pumping field $h_{1}$ at fixed $\mu_{0} H_{0}=0.52 \mathrm{~T}$. (f) Nutation spectroscopy map at fixed $h_{1}$ of the small amplitude dynamics $\Delta M_{2}$ excited by $\omega_{2}$ as a function of the down-swept field $H_{0}$. (g) Simultaneous measurement of $\Delta M_{1}$ induced by the main pumping at $\omega_{1}$. (h,i) Same as $(f, g)$ for a smaller main pumping power. In panels (e)-(i), the black dashed lines show the analytical predictions from Eq. (1), and the red dotted lines show the results from micromagnetic simulations.

is a minimum amplitude of $\mu_{0} h_{1} \simeq 0.15 \mathrm{mT}$ to drive the $\mathbf{P}$ mode at $\mu_{0} H_{0}=0.52 \mathrm{~T}$, which corresponds to a normalized foldover shift $\left(H_{K}-H_{0}\right) / M_{s}=23 \%$, i.e., an angle of precession $\theta_{0} \simeq 40^{\circ}$. Above this amplitude, the nutation frequency is defined, and it increases with $h_{1}$ as predicted by Eq. (1), which demonstrates that the nonlinear demagnetizing term is essential to describe the nutation dynamics. To illustrate this, we have plotted, using blue dotted lines, the behavior predicted by Eq. (1) in its absence, which underestimates by about an order of magnitude the observed nutation frequency.

We now interpret the nutation spectroscopy maps of Figs. 2(f) and 2(h), where $\Delta M_{2}$ is measured at fixed $h_{1}$ by sweeping $H_{0}$ and $\omega_{2}$. When $H_{0}>H_{K}$, the magnetization dynamics is driven off resonantly by $\boldsymbol{h}_{1}$ and has small amplitude. Hence, the weak microwave field $\boldsymbol{h}_{2}$ simply excites the linear Kittel resonance on top of it, which explains the bright linear dispersion $\omega_{2}=\gamma \mu_{0}\left(H_{0}-M_{s}\right)$ observed in this region. This result is also expected from Eq. (1), which in the limit of small $\theta_{0}$, tends towards the difference between the Kittel frequency of small linear oscillations $\omega_{K}$ and the frequency of the microwave field $\omega_{1}$. The situation is quite different when $H_{0}<H_{K}$. In this case, $\boldsymbol{h}_{1}$ drives the strong foldover regime demonstrated in Fig. 1 , thereby appreciably changing both $\theta_{0}$ and $\varphi_{0}$ upon sweeping down $H_{0}$, and $\boldsymbol{h}_{2}$ excites the magnetization dynamics on top of the corresponding $\mathbf{P}$-mode. The nontrivial evolution of the two resonance branches symmetrically distributed around the main pumping frequency $\omega_{1}$ as a function of $H_{0}$ is reproduced qualitatively by Eq. (1), and quantitatively by micromagnetic simulations. The fact that the upper branch is continuous with the linear Kittel resonance branch observed above $H_{K}$ indicates that the perturbation of the $\mathbf{P}$-mode driven by $\boldsymbol{h}_{2}$ has a uniform phase, i.e., corresponds to a uniform nutation of the magnetization.

The experimental results presented in Fig. 2 also demonstrate that the weak resonant excitation of the nutation mode can destabilize the strong foldover dynamics. Figures 2(g) and 2(i) display the evolution of $\Delta M_{1}$ induced by the cw pumping $\boldsymbol{h}_{1}$ at $\omega_{1}$ while exciting the nutation dynamics with $\boldsymbol{h}_{2}$ as a function of $H_{0}$ and $\omega_{2}$, whose $\Delta M_{2}$ spectroscopy is presented in Figs. 2(f) and 2(h), respectively. In these $2 \mathrm{D}$ maps, the foldover breakdown occurring at $H_{\text {down }}$ is easily identified thanks to the associated sharp change of $\Delta M_{1}$, and it is anticipated as the nutation mode is excited. It is particularly clear in Fig. 2(i), where the maximal foldover shift $\mu_{0}\left(H_{K}-H_{\text {down }}\right)$ is reduced by almost $0.05 \mathrm{~T}$ when $\left|\omega_{2}-\omega_{1}\right| /(2 \pi) \simeq 0.2 \mathrm{GHz}$, which corresponds to the nutation resonance. Moreover, these data suggest that higher-order nutation modes can be excited by $\boldsymbol{h}_{2}$ since an anticipated foldover breakdown is also observed at $\left|\omega_{2}-\omega_{1}\right| /(2 \pi) \simeq 0.35 \mathrm{GHz}$.

In order to investigate these other nutation modes, we perform the same measurements as in Figs. 2(f)-2(i) but for larger detunings $\omega_{2}-\omega_{1}\left(P_{2}\right.$ is also increased from -19 to $-13 \mathrm{dBm})$. The results obtained at $P_{1}=+1 \mathrm{dBm}$ are reported in Figs. 3(b) and 3(c) (those obtained at $P_{1}=$ $+8 \mathrm{dBm}$ are presented in the Supplemental Material, Fig. S2 [26]). The spectroscopy map of the SW modes excited by $\boldsymbol{h}_{2}$ in the absence of $\boldsymbol{h}_{1}$ is shown in Fig. 3(a). It displays the linear dispersion relation of the radial SW modes excited by the uniform field $\boldsymbol{h}_{2}$, already discussed in Fig. 1(c). Because of the strong foldover regime driven by $\boldsymbol{h}_{1}$ at $H_{0}<H_{K}$ in Fig. 3(b), each of these radial SW branches transforms into a pair of branches symmetric around $\omega_{1}$. Additionally, there is a pair of branches that appears at twice the main nutation frequency, which is due to the ellipticity of the trajectory, apparent in Fig. 2(c). The macrospin approach used to derive Eq. (1) cannot be used to account for these higher-order nutation modes, although plane-wave perturbations to the $\mathbf{P}$-mode can also be analytically calculated [39]. We therefore use micromagnetic simulations to calculate the SW nutation spectra shown in Fig. 3(d), which are in good agreement with the experiments. The extracted SW nutation mode profiles (see Appendix C), shown in the insets of Fig. 3(d), indicate that there is some continuity between the radial SW modes excited in the linear regime on top of the equilibrium magnetization and the nutation modes excited on top of the $\mathbf{P}$-mode driven in the nonlinear regime by $\boldsymbol{h}_{1}$. Finally, Fig. 3(c) confirms that the excitation of the nutation resonances can destabilize the strong foldover dynamics, with sizable reductions of the switching field $H_{\text {down }}$ observed at the corresponding nutation frequencies. 

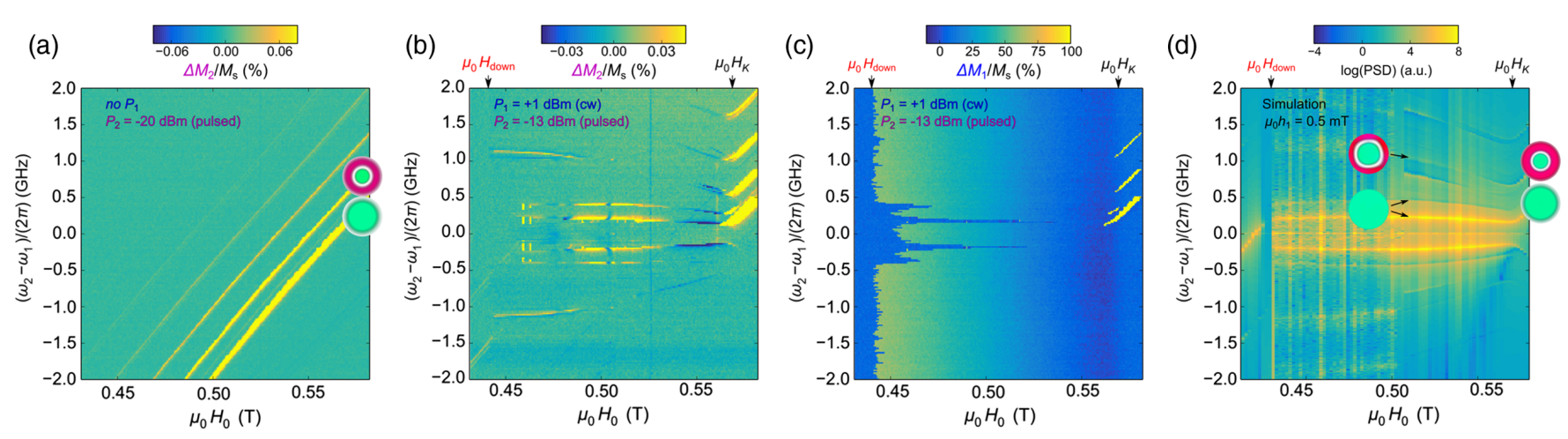

FIG. 3. Spin-wave nutation modes. (a) Spectroscopy map of the SW modes excited by the low power excitation at $\omega_{2}$ in the absence of the main pumping at $\omega_{1} /(2 \pi)=10.5 \mathrm{GHz}$. They correspond to the same radial modes as probed in the linear regime in Fig. $1 \mathrm{c}$, whose profiles are recalled as insets. (b) $\Delta M_{2}$ spectroscopy map of the SW nutation modes excited by the low power excitation at $\omega_{2}$ in the presence of the main excitation at $\omega_{1}\left(P_{1}=+1 \mathrm{dBm}\right)$. (c) Simultaneous measurement of $\Delta M_{1}$ induced by the main pumping at $\omega_{1}$. (d) Micromagnetic simulations of the experimental data shown in (b). The SW mode profiles shown as insets are extracted at some specific $\omega_{2}-\omega_{1}$ and $H_{0}$. In all the panels, the perpendicular field $H_{0}$ is swept down.

\section{DISCUSSION}

As in the case of a spinning top, the nutation of magnetization demonstrated above is made possible by the specific properties of the dynamics on the unit sphere [2]. Namely, it is topologically allowed for the magnetization to oscillate around its fixed point $\boldsymbol{M}_{0}\left(\theta_{0}, \varphi_{0}\right)$ in the rotating frame, which is set by the drive $\boldsymbol{h}_{1}$. The nutation dynamics discussed in this work is thus fundamentally connected to the existence of the $\mathbf{P}$-mode. The nutation frequency results from the balance of torques acting on the angular momentum and is given by Eq. (1) in the case of a macrospin governed by the LLG equation. The accuracy of the latter to account for the experimental data means that the coherent precession of the magnetization vector is indeed dominating the deeply nonlinear driven dynamics, despite the signatures of SW instabilities observed at very large pumping power.

This preservation of coherent magnetization dynamics, which we report on a thin disc with submicrometric diameter, is mainly due to the geometric confinement. It significantly reduces the density of normal modes and suppresses the nonlinear SW interactions present in bulk ferromagnets $[31,32]$. In addition, in our experiments, the ground state has magnetization perpendicular to the plane. In this case, the uniform mode in thin films lies at the bottom of the SW manifold; thus, it has no degenerate mode to couple to $[40,41]$, and the angle of the purely circular precession driven by FMR can reach large values [42]. These combined circumstances allow for the excitation of largeamplitude quasiuniform precession of magnetization. The main effect of the SW instabilities, which eventually occur at very large pumping power, is to slightly reduce the nutation frequency, which is well captured by full micromagnetic simulations. This effect can be ascribed to the shift of the phase between the pumping field and the average magnetization [43] observed in our simulations, a key effect to explain the above threshold dynamics [7,44]. The nutation spectroscopy of magnetization thus allows a more detailed investigation of the highly nonlinear regime, where autooscillation instabilities $[6,40,45,46]$ and instability patterns [47] have been evidenced.

Our results also highlight that the dynamical states driven by a high-power microwave signal can be controlled using a second signal with much lower power by the resonant excitation of the nutation modes. This case could be applied in devices taking advantage of the bistable magnetization dynamics for microwave signal processing [27], in analogy to microwave-assisted magnetization switching [48-50]. Furthermore, the frequency selectivity and high susceptibility of nutation excitations provide new potentials for the scheme of neuromorphic computing. Cognitive tasks have already been implemented using the nonlinear dynamics of nanomagnets, from the transient regime of a single STNO [17] to the collective behavior of mutually coupled STNOs controlled by external microwave signals [18,51]. An appropriate use of the nutation dynamics of magnetization would allow us to gain additional control on nonlinear dynamics, which is highly desired in this field.

\section{ACKNOWLEDGMENTS}

Y.L. thanks the French National Research Agency for funding under Contract No. ANR-14-CE260021 (MEMOS), V.V.N. acknowledges the Russian Competitive Growth program of Kazan Federal University, and C. S. acknowledges the Labex NanoSaclay (ANR-10-LABX-0035) for support under the action "Rayonnement International." This research was partially funded by the ANR-18-CE24-0021 (MAESTRO) project.

\section{APPENDIX A: EXPERIMENTAL DETAILS}

\section{Sample preparation}

A 20-nm-thick $\mathrm{Y}_{3} \mathrm{Fe}_{5} \mathrm{O}_{12}$ (YIG) film was grown by pulsed laser deposition on a (111) $\mathrm{Gd}_{3} \mathrm{Ga}_{5} \mathrm{O}_{12}$ (GGG) substrate, as described in Ref. [22]. It was used to pattern 
the studied YIG nanodisc by electron lithography and dry etching. After the insertion of a 50-nm-thick $\mathrm{SiO}_{2}$ insulating layer, a $150-\mathrm{nm}$-thick and 5- $\mu \mathrm{m}$-wide gold antenna was defined on top of the nanodisc to provide the microwave excitation [25].

\section{MRFM setup}

The magnetic resonance force microscope is located between the poles of an electromagnet and operated under vacuum $\left(10^{-6} \mathrm{mbar}\right)$ at a stabilized temperature of $288 \mathrm{~K}$. The cantilever is an Olympus Biolever (spring constant $5 \mathrm{mN} \cdot \mathrm{m}^{-1}$ ) with a 700-nm-diameter sphere of an amorphous FeSi alloy (magnetic moment $0.28 \mathrm{pA} \cdot \mathrm{m}^{2}$ ) glued to its apex. In this study, MRFM spectroscopy is achieved by placing the center of this magnetic nanosphere at a distance of 1.5-1.8 $\mu \mathrm{m}$ above the center of the YIG nanodisc. The stray field of the MRFM probe (10-16 mT) is subtracted from the corresponding spectra. The displacement of the cantilever is monitored using optical techniques. Its mechanical frequency $\left(f_{c} \approx 12.3 \mathrm{kHz}\right)$ is tracked using a phase-locked loop, and its vibration amplitude is stabilized to $4 \mathrm{~nm}$ using a piezoelectric bimorph. When the $\mathrm{cw}$ microwave pumping excites the magnetization dynamics in the sample, its longitudinal component is reduced, so the static dipolar force with the magnetic probe diminishes. The associated variation of the cantilever frequency provides a quantitative magnetometry of the sample [52]. In order to improve the signal-to-noise ratio, the microwave excitation is pulsed on and off at $f_{c}$. In that case, the cantilever vibrations induced by the magnetization dynamics excited in the sample are enhanced by the quality factor $Q \approx 2000$ of the mechanical detection [23].

\section{Microwave field calibration}

We use the onset of foldover as a means to calibrate the amplitude of the excitation field produced by the microwave antenna at the sample location [24]. At the threshold of foldover instability, $h_{1}^{\text {th }}=0.62 \Delta H^{3 / 2} / M_{s}^{1 / 2}[20]$, and the slope of the FMR curve becomes infinite on the low field side of the resonance, which is experimentally observed at $10.5 \mathrm{GHz}$ for an output power from the synthesizer $P_{1}^{\text {th }}=-33 \mathrm{dBm}$. Using the FMR linewidth measured in the linear regime, one gets $\mu_{0} h_{1}^{\text {th }}=0.009 \mathrm{mT}$, i.e., a calibration factor $a=0.4 \mathrm{mT} / \sqrt{\mathrm{mW}}$ between microwave field and power. To get a better precision, we also fit the dependence on power of the critical fields $H_{\text {down }}$ and $H_{\text {up }}$ determined experimentally beyond the foldover onset [28], which yields $a=0.41 \pm 0.03 \mathrm{mT} / \sqrt{\mathrm{mW}}$.

\section{APPENDIX B: ANALYTICAL CALCULATIONS}

The nonlinear FMR excited in a uniaxial system by the superposition of two time-harmonic external fields, $\boldsymbol{h}_{\mathrm{ac}}(t)=\boldsymbol{h}_{1}(t)+\boldsymbol{h}_{2}(t)$ with $\left|\boldsymbol{h}_{2}(t)\right| \ll\left|\boldsymbol{h}_{1}(t)\right|$, is calculated based on the macrospin LLG equation. The main stages of the analytical derivation presented in the Supplemental Material [26] are the following. The LLG equation is first written in the frame of reference rotating around the $z$ axis at the angular frequency $\omega_{1}$ of the dominant time-harmonic component: $\dot{\boldsymbol{m}}-\alpha \boldsymbol{m} \times \dot{\boldsymbol{m}}=-\boldsymbol{m} \times\left(\boldsymbol{h}_{\text {eff }}-\omega_{1} \boldsymbol{e}_{z}\right)+\alpha \omega_{1} \boldsymbol{m} \times$ $\left(\boldsymbol{e}_{z} \times \boldsymbol{m}\right)$, where $\boldsymbol{h}_{\text {eff }}=\kappa_{\mathrm{eff}} m_{z} \boldsymbol{e}_{z}+h_{0} \boldsymbol{e}_{z}+\boldsymbol{h}_{\mathrm{ac}}(t), \dot{\boldsymbol{m}}$ is the time derivative of the normalized magnetization vector taken in the rotating frame, $\boldsymbol{h}_{0}$ is the normalized bias field, $\boldsymbol{e}_{z}$ is the unit vector along it, and $\kappa_{\text {eff }}$ is the effective anisotropy constant. It is then written in spherical coordinates and considered in the case where only the right circularly polarized component of $\boldsymbol{h}_{1}$ is applied. This method allows us to find its equilibrium points $\boldsymbol{m}_{0}$ (P-modes), or equivalently $\left(\theta_{0}, \varphi_{0}\right)$, and to analyze the foldover of FMR [33]. The standard analysis of the stability of these P-modes allows us to calculate the nutation frequency given in Eq. (1) [39]. In brief, $\boldsymbol{m}$ is expanded around $\boldsymbol{m}_{0}$ to linearize the LLG equation, and the complex amplitudes of magnetization perturbations are calculated in the rotating frame by projecting them in the plane $\left(\boldsymbol{e}_{1}, \boldsymbol{e}_{2}\right)$ orthogonal to $\boldsymbol{m}_{0}$, where $\sin \theta_{0} \boldsymbol{e}_{1}=$ $\left(\boldsymbol{e}_{z} \times \boldsymbol{m}_{0}\right) \times \boldsymbol{m}_{0}$ and $\sin \theta_{0} \boldsymbol{e}_{2}=\left(\boldsymbol{e}_{z} \times \boldsymbol{m}_{0}\right)$. Finally, the P-mode linear response to the small additional microwave field $\boldsymbol{h}_{2}$ is studied.

\section{APPENDIX C: MICROMAGNETIC SIMULATIONS}

The magnetization dynamics in the YIG nanodisc is calculated using the PYTHON module MicroMagnum, a micromagnetic finite difference simulator that can be run on graphics processing unit [53]. The nominal geometry of the nanodisc (diameter $700 \mathrm{~nm}$, thickness $20 \mathrm{~nm}$ ) is discretized using a $128 \times 128 \times 1$ rectangular mesh. The following magnetic parameters are used: $M_{s}=167 \mathrm{kA} \cdot \mathrm{m}^{-1}$, $A_{\mathrm{ex}}=4.3 \mathrm{p} \mathrm{J} \cdot \mathrm{m}^{-1}$ (exchange length $\simeq 16 \mathrm{~nm}$ ), $\gamma /(2 \pi)=$ $28.5 \mathrm{GHz} \cdot \mathrm{T}^{-1} \cdot \mathrm{s}^{-1}$, and $\alpha=5 \times 10^{-4}$. The bias magnetic field is applied along the normal of the disc. The static equilibrium configuration of the magnetization is calculated at $0.59 \mathrm{~T}$. Then, a linearly polarized excitation field of constant amplitude is applied at $10.5 \mathrm{GHz}$ in the plane of the disc, and for each value of the bias magnetic field, which is decreased by steps of $0.002 \mathrm{~T}$, the resulting magnetization dynamics is calculated over $100 \mathrm{~ns}$ with a typical step of $3 \mathrm{ps}$. This process allows us to reproduce the foldover regime demonstrated in the experiments and to calculate the nutation frequencies. Those frequencies are obtained by fast Fourier transformation of the transient dynamics of the average magnetization simulated at each bias field. The nutation mode profiles are obtained by stroboscopically averaging the magnetization dynamics at the corresponding nutation frequencies in the rotating frame. 
[1] C. Kittel, Introduction to Solid State Physics, 8th ed. (Wiley, New York, 2004).

[2] I. D. Mayergoyz, G. Bertotti, and C. Serpico, Nonlinear Magnetization Dynamics in Nanosystems (Elsevier, New York, 2009).

[3] A. G. Gurevich and G. A. Melkov, Magnetization Oscillations and Waves (CRC Press, Boca Raton, FL, 1996).

[4] Nonlinear Phenomena and Chaos in Magnetic Materials, edited by P.E. Wigen (World Scientific, Singapore, 1994).

[5] S. M. Mohseni, S. R. Sani, J. Persson, T. N. A. Nguyen, S. Chung, Y. Pogoryelov, P. K. Muduli, E. Iacocca, A. Eklund, R. K. Dumas, S. Bonetti, A. Deac, M. A. Hoefer, and J. Akerman, Spin Torque-Generated Magnetic Droplet Solitons, Science 339, 1295 (2013).

[6] S. M. Rezende and F. M. de Aguiar, Spin-Wave Instabilities, Auto-Oscillations, and Chaos in Yttrium-Iron-Garnet, Proc. IEEE 78, 893 (1990).

[7] V. S. L'vov, Wave Turbulence under Parametric Excitation: Applications to Magnets (Springer-Verlag, Berlin, 1994).

[8] S. Petit-Watelot, J.-V. Kim, A. Ruotolo, R. M. Otxoa, K. Bouzehouane, J. Grollier, A. Vansteenkiste, B. Van de Wiele, V. Cros, and T. Devolder, Commensurability and Chaos in Magnetic Vortex Oscillations, Nat. Phys. 8, 682 (2012).

[9] S. O. Demokritov, V. E. Demidov, O. Dzyapko, G. A. Melkov, A. A. Serga, B. Hillebrands, and A. N. Slavin, Bose-Einstein Condensation of Quasi-equilibrium Magnons at Room Temperature under Pumping, Nature (London) 443, 430 (2006).

[10] A. V. Chumak, V. I. Vasyuchka, A. A. Serga, and B. Hillebrands, Magnon Spintronics, Nat. Phys. 11, 453 (2015).

[11] S. I. Kiselev, J. C. Sankey, I. N. Krivorotov, N. C. Emley, R. J. Schoelkopf, R. A. Buhrman, and D. C. Ralph, Microwave Oscillations of a Nanomagnet Driven by a SpinPolarized Current, Nature (London) 425, 380 (2003).

[12] D. Houssameddine, U. Ebels, B. Delat, B. Rodmacq, I. Firastrau, F. Ponthenier, M. Brunet, C. Thirion, J.-P. Michel, L. Prejbeanu-Buda, M.-C. Cyrille, O. Redon, and B. Dieny, Spin-Torque Oscillator Using a Perpendicular Polarizer and a Planar Free Layer, Nat. Mater. 6, 447 (2007).

[13] W. Chen, G. de Loubens, J.-M. L. Beaujour, J. Z. Sun, and A. D. Kent, Spin-Torque Driven Ferromagnetic Resonance in a Nonlinear Regime, Appl. Phys. Lett. 95, 172513 (2009).

[14] A. Hamadeh, G. de Loubens, V. V. Naletov, J. Grollier, C. Ulysse, V. Cros, and O. Klein, Autonomous and Forced Dynamics in a Spin-Transfer Nano-Oscillator: Quantitative Magnetic-Resonance Force Microscopy, Phys. Rev. B 85, 140408(R) (2012).

[15] M. Collet, X. de Milly, O. d'Allivy Kelly, V. V. Naletov, R. Bernard, P. Bortolotti, J. Ben Youssef, V. E. Demidov, S. O. Demokritov, J. L. Prieto, M. Muñoz, V. Cros, A. Anane, G. de Loubens, and O. Klein, Generation of Coherent SpinWave Modes in Yttrium Iron Garnet Microdiscs by SpinOrbit Torque, Nat. Commun. 7, 10377 (2016).

[16] A. Slavin and V. Tiberkevich, Nonlinear Auto-Oscillator Theory of Microwave Generation by Spin-Polarized Current, IEEE Trans. Magn. 45, 1875 (2009).
[17] J. Torrejon, M. Riou, F. A. Araujo, S. Tsunegi, G. Khalsa, D. Querlioz, P. Bortolotti, V. Cros, K. Yakushiji, A. Fukushima, H. Kubota, S. Yuasa, M. D. Stiles, and J. Grollier, Neuromorphic Computing with Nanoscale Spintronic Oscillators, Nature (London) 547, 428 (2017).

[18] M. Romera, P. Talatchian, S. Tsunegi, F. A. Araujo, V. Cros, P. Bortolotti, J. Trastoy, K. Yakushiji, A. Fukushima, H. Kubota et al., Vowel Recognition with Four Coupled SpinTorque Nano-Oscillators, Nature (London) 563, 230 (2018).

[19] H. Suhl, The Theory of Ferromagnetic Resonance at High Signal Powers, J. Phys. Chem. Solids 1, 209 (1957).

[20] P. W. Anderson and H. Suhl, Instability in the Motion of Ferromagnets at High Microwave Power Levels, Phys. Rev. 100, 1788 (1955).

[21] A. A. Serga, A. V. Chumak, and B. Hillebrands, YIG Magnonics, J. Phys. D 43, 264002 (2010).

[22] O. d'Allivy Kelly, A. Anane, R. Bernard, J. B. Youssef, C. Hahn, A. H. Molpeceres, C. Carretero, E. Jacquet, C. Deranlot, P. Bortolotti, R. Lebourgeois, J.-C. Mage, G. de Loubens, O. Klein, V. Cros, and A. Fert, Inverse Spin Hall Effect in Nanometer-Thick Yttrium Iron Garnet/Pt System, Appl. Phys. Lett. 103, 082408 (2013).

[23] O. Klein, G. de Loubens, V. V. Naletov, F. Boust, T. Guillet, H. Hurdequint, A. Leksikov, A. N. Slavin, V. S. Tiberkevich, and N. Vukadinovic, Ferromagnetic Resonance Force Spectroscopy of Individual Submicron-Size Samples, Phys. Rev. B 78, 144410 (2008).

[24] V. V. Naletov, G. de Loubens, G. Albuquerque, S. Borlenghi, V. Cros, G. Faini, J. Grollier, H. Hurdequint, N. Locatelli, B. Pigeau, A. N. Slavin, V. S. Tiberkevich, C. Ulysse, T. Valet, and O. Klein, Identification and Selection Rules of the SpinWave Eigenmodes in a Normally Magnetized Nanopillar, Phys. Rev. B 84, 224423 (2011).

[25] C. Hahn, V. V. Naletov, G. de Loubens, O. Klein, O. d'Allivy Kelly, A. Anane, R. Bernard, E. Jacquet, P. Bortolotti, V. Cros, J. L. Prieto, and M. Muñoz, Measurement of the Intrinsic Damping Constant in Individual Nanodisks of $\mathrm{Y}_{3} \mathrm{Fe}_{5} \mathrm{O}_{12}$ and $\mathrm{Y}_{3} \mathrm{Fe}_{5} \mathrm{O}_{12} \mid \mathrm{Pt}$, Appl. Phys. Lett. 104, 152410 (2014).

[26] See Supplemental Material at [http://link.aps.org/ supplemental/10.1103/PhysRevX.9.041036 will be inserted by publisher] for supplementary figures and detailed analytical derivations.

[27] Y. K. Fetisov, C. E. Patton, and V. T. Synogach, Nonlinear Ferromagnetic Resonance and Foldover in Yttrium Iron Garnet Thin Films-Inadequacy of the Classical Model, IEEE Trans. Magn. 35, 4511 (1999).

[28] Y. S. Gui, A. Wirthmann, and C.-M. Hu, Foldover Ferromagnetic Resonance and Damping in Permalloy Microstrips, Phys. Rev. B 80, 184422 (2009).

[29] G. de Loubens, V. V. Naletov, and O. Klein, Reduction of the Spin-Wave Damping Induced by Nonlinear Effects, Phys. Rev. B 71, 180411(R) (2005).

[30] V. V. Naletov, V. Charbois, O. Klein, and C. Fermon, Quantitative Measurement of the Ferromagnetic Resonance Signal by Force Detection, Appl. Phys. Lett. 83, 3132 (2003).

[31] Y. Kobljanskyj, G. Melkov, K. Guslienko, V. Novosad, S. D. Bader, M. Kostylev, and A. Slavin, Nano-Structured 
Magnetic Metamaterial with Enhanced Nonlinear Properties, Sci. Rep. 2, 478 (2012).

[32] G. A. Melkov, D. V. Slobodianiuk, V. S. Tiberkevich, G. de Loubens, O. Klein, and A. N. Slavin, Nonlinear Ferromagnetic Resonance in Nanostructures Having Discrete Spectrum of Spin-Wave Modes, IEEE Magn. Lett. 4, 4000504 (2013).

[33] G. Bertotti, C. Serpico, and I. D. Mayergoyz, Nonlinear Magnetization Dynamics under Circularly Polarized Field, Phys. Rev. Lett. 86, 724 (2001).

[34] A. Abragam, Principles of Nuclear Magnetism (Oxford University Press, Oxford, 1961).

[35] A. Capua, C. Rettner, S.-H. Yang, T. Phung, and S. S. P. Parkin, Ensemble-Averaged Rabi Oscillations in a Ferromagnetic CoFeB Film, Nat. Commun. 8, 16004 (2017).

[36] M.-C. Ciornei, J. M. Rubí, and J.-E. Wegrowe, Magnetization Dynamics in the Inertial Regime: Nutation Predicted at Short Time Scales, Phys. Rev. B 83, 020410(R) (2011).

[37] E. Olive, Y. Lansac, and J.-E. Wegrowe, Beyond Ferromagnetic Resonance: The Inertial Regime of the Magnetization, Appl. Phys. Lett. 100, 192407 (2012).

[38] Y. Li, A.-L. Barra, S. Auffret, U. Ebels, and W. E. Bailey, Inertial Terms to Magnetization Dynamics in Ferromagnetic Thin Films, Phys. Rev. B 92, 140413(R) (2015).

[39] G. Bertotti, I. D. Mayergoyz, and C. Serpico, Spin-Wave Instabilities in Large-Scale Nonlinear Magnetization Dynamics, Phys. Rev. Lett. 87, 217203 (2001).

[40] R. D. McMichael and P. E. Wigen, High-Power Ferromagnetic Resonance without a Degenerate Spin-Wave Manifold, Phys. Rev. Lett. 64, 64 (1990).

[41] Yu. V. Gulyaev, P. E. Zil'berman, A. G. Temiryazev, and M. P. Tikhomirova, Principal Mode of the Nonlinear SpinWave Resonance in Perpendicular Magnetized Ferrite Films, Phys. Solid State 42, 1094 (2000).

[42] K. Gnatzig, H. Dötsch, M. Ye, and A. Brockmeyer, Ferrimagnetic Resonance in Garnet Films at Large Precession Angles, J. Appl. Phys. 62, 4839 (1987).

[43] H. G. Bauer, P. Majchrak, T. Kachel, C. H. Back, and G. Woltersdorf, Nonlinear Spin-Wave Excitations at Low Magnetic Bias Fields, Nat. Commun. 6, 8274 (2015).
[44] T. Gerrits, P. Krivosik, M. L. Schneider, C. E. Patton, and T. J. Silva, Direct Detection of Nonlinear Ferromagnetic Resonance in Thin Films by the Magneto-Optical Kerr Effect, Phys. Rev. Lett. 98, 207602 (2007).

[45] B. Lührmann, M. Ye, H. Dötsch, and A. Gerspach, Nonlinearities in the Ferrimagnetic Resonance in Epitaxial Garnet Films, J. Magn. Magn. Mater. 96, 237 (1991).

[46] S. Watanabe, D. Hirobe, Y. Shiomi, R. Iguchi, S. Daimon, M. Kameda, S. Takahashi, and E. Saitoh, Generation of Megahertz-Band Spin Currents Using Nonlinear Spin Pumping, Sci. Rep. 7, 4576 (2017).

[47] R. Bonin, M. d'Aquino, G. Bertotti, C. Serpico, and I. D. Mayergoyz, Analysis of Magnetization Instability Patterns in Spin-Transfer Nano-Oscillators, Eur. Phys. J. B 85, 47 (2012).

[48] C. Thirion, W. Wernsdorfer, and D. Mailly, Switching of Magnetization by Nonlinear Resonance Studied in Single Nanoparticles, Nat. Mater. 2, 524 (2003).

[49] B. Pigeau, G. de Loubens, O. Klein, A. Riegler, F. Lochner, G. Schmidt, and L. W. Molenkamp, Optimal Control of Vortex-Core Polarity by Resonant Microwave Pulses, Nat. Phys. 7, 26 (2011).

[50] H. Suto, T. Kanao, T. Nagasawa, K. Mizushima, and R. Sato, Magnetization Switching of a $\mathrm{Co} / \mathrm{Pt}$ Multilayered Perpendicular Nanomagnet Assisted by a Microwave Field with Time-Varying Frequency, Phys. Rev. Applied 9, 054011 (2018).

[51] Y. Li, X. de Milly, F. A. Araujo, O. Klein, V. Cros, J. Grollier, and G. de Loubens, Probing Phase Coupling between Two Spin-Torque Nano-Oscillators with an External Source, Phys. Rev. Lett. 118, 247202 (2017).

[52] H. Lavenant, V. Naletov, O. Klein, G. de Loubens, L. Casado, and J. M. De Teresa, Mechanical Magnetometry of Cobalt Nanospheres Deposited by Focused Electron Beam at the Tip of Ultra-Soft Cantilevers, Nanofabrication 1, 65 (2014).

[53] See http://micromagnum.informatik.uni-hamburg.de. 